\title{
Steel Corrosion Assessment by Electrochemical Impedance on Metakaolin Blended Mortars
}

\author{
Víctor Triana ${ }^{\mathrm{a}}$, Juan Lizarazo-Marriaga ${ }^{\mathrm{a}}{ }^{\text {, Jhon Olaya Flórez }}{ }^{\mathrm{b}}$ \\ ${ }^{\mathrm{a}}$ Materials and Structures Research Group, Universidad Nacional de Colombia, \\ Ciudad Universitaria, Bogotá, Colombia \\ ${ }^{\mathrm{b}}$ Mechanical Engineering Departament, Universidad Nacional de Colombia, \\ Ciudad Universitaria, Bogotá, Colombia
}

Received: October 10, 2012; Revised: September 27, 2013

\begin{abstract}
Since Corrosion of reinforcing steel in concrete is the cause of major economic losses, Portland cement has been traditionally replaced by cements blended with pozzalanic materials, most of which have been found to reduce the corrosion of steel. This paper shows the results of an experimental research aimed to investigate the corrosion of reinforcement in mortar using electrochemical impedance spectroscopy (EIS). For this, concrete laboratory samples containing a $0.0055 \mathrm{~m}$ steel bar and prepared with just ordinary Portland cement (OPC) and metakaolin at a replacement level of $20 \%$ were analyzed. In order to accelerate the steel corrosion process, all the samples were kept in a 3\% $\mathrm{NaCl}$ solution and a constant anodic electrical potential was applied. Variations in the water to cementitious material ratio $(0.5$ and 0.6$)$ and metakaolin proportion were analyzed, while the cementitious material to sand ratio was kept constant at 1:2.25 in all of the specimens. The results showed a reduction in corrosion rates when metakaolin was used as a blending admixture, especially at water to cementitious material ratio of 0.5 .
\end{abstract}

Keywords: electrochemical impedance spectroscopy, pozzolanic materials, metakaolin, reinforcement corrosion

\section{Introduction}

Corrosion is defined as the deterioration of a material by a chemical or electrochemical action in the environment surrounding it. Therefore, corrosion is a natural process in which a metallic element is transformed into a more stable compound, usually an oxide ${ }^{1}$. A study carried out by the United States government has estimated the total direct costs incurred by corrosion issues to be approximately US $\$ 276$ billion per year ${ }^{2}$, which represents $3.1 \%$ of the country's gross domestic product. That study has revealed that US $\$ 50$ billion of these costs are related to the construction industry, representing $18.1 \%$ of the total corrosion costs incurred in the United States. The losses due to the corrosion of reinforcing steel in concrete structures have been also included in this percentage. Concrete is the most widely used material in construction due to its attractive physical and mechanical properties and its economic advantages over other materials. For these reasons, reinforced concrete has been the topic of several research projects aimed at increasing its resistance to corrosion by replacing industrial by-products or natural pozzolanic materials. These mineral admixtures are used to reduce costs and enhance the properties of concrete, particularly its durability, which can also help reduce corrosion of the reinforcement, and they are also more environmentally friendly because they are composed of industrial waste or simply require less energy to be fabricated than conventional Portland cement. One of

*e-mail: jmlizarazom@unal.edu.co these materials is metakaolin (MK), an artificial pozzolanic material produced from the calcination of natural kaolin at temperatures between $500^{\circ} \mathrm{C}$ and $800^{\circ} \mathrm{C}$, which transforms its crystalline structure and results in a thermally activated aluminosilicate. This material is used as a supplementary binder at replacement levels between 15 and 35\% in both, mortar and concrete ${ }^{3}$.

Most of the existing research on blended concrete has been focused on enhancing its durability to protect against the penetration of damaging agents that can cause corrosion, and on studying the material without considering the behavior of reinforcing steel. However, electrochemical techniques such as linear polarization and electrochemical impedance spectroscopy (EIS) have begun to be used in some recent concrete research. Although these techniques have previously been employed mainly in the study of corrosion mechanisms in metals and coatings, it is possible to determine the behavior of both, concrete and reinforcing steel ${ }^{4-8}$.

With these issues in mind, this paper is focused on the study of corrosion phenomena in mortars blended with metakaolin using electrochemical impedance spectroscopy. The influence of concrete's water to cementitious material ratio $(0.5-0.6)$ and the replacement of metakaolin $(0 \%-20 \%)$ were investigated in order to determine the corrosion rate of reinforcing steel, which was analyzed using principally EISNyquist diagrams. Additionally, standard RCPT (ASTM C1202) and compressive strength tests were carried out. 


\section{Experimental Programme}

Impedance describes electrical resistance and is used in alternating current circuits. EIS is based on the use of a current signal that is applied at different frequencies over an electrode (corroding metal) to determine the corresponding response. The relationship between the impedance and applied frequency can be obtained from the impedance spectra or obtained theoretically using the "equivalent electrical circuits". These circuits are composed of elements such as resistors, capacitors, and inductors ${ }^{9}$. The EIS is useful because its results are expressed as a function of time, so that when frequencies are applied in certain ranges, the sinusoidal wave is so rapid that it passes through the medium as if it were in a short circuit ${ }^{6}$. When applying a sinusoidal excitation potential with low amplitude within a frequency range, the current response will also have a sinusoidal form, but it will be dephased and have different amplitude. Figure 1 illustrates this behavior. An analogy with direct current is described as the limit at which the frequency tends to become zero. In other words, as the dephasing angle between the potential and the current tends to become zero, the impedance $\mathrm{Z}$ becomes more similar to the resistance $\mathrm{R}$ (ohms) given by Ohm's law for direct current.

The information obtained from EIS tests are described by the Nyquist and Bode diagrams. The Nyquist spectra corresponds to the relationship between the resistivity, Z' (real impedance), and the capacitance, Z' (imaginary impedance). The Bode diagrams are represented by plots of the logarithm of the frequency versus the logarithm of the impedance modulus and the phase angle. These plots provide information about the electrochemical behavior of a system in different environments, which was of major importance in this study on concrete durability. For example, it is possible to simultaneously obtain the behavior of the coating matrix (concrete, mortar or paste) and the response of the reinforcing steel. This paper shows the results of mortar mixes tested after 495 days of casting, measured in 3 identical samples with the goal of avoiding the inherent variability of the test procedure.

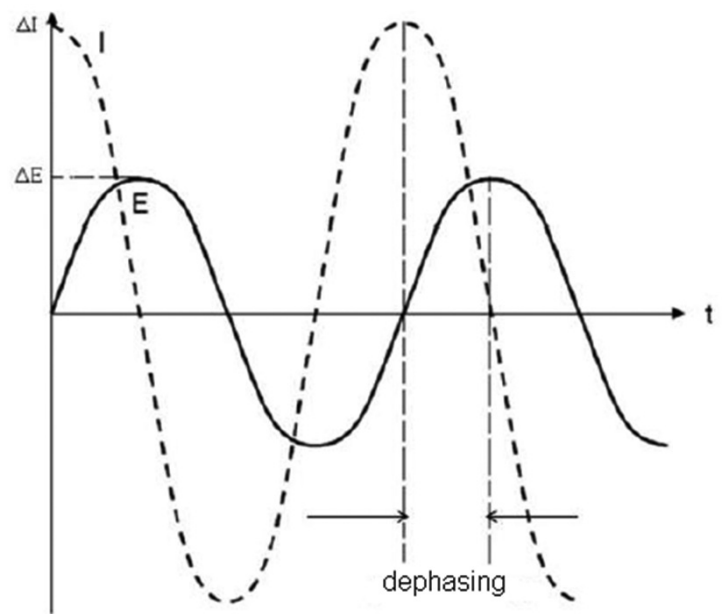

Figure 1. Dephased Current (I) and potential (E) as function of time (t) in an AC circuit.
Additionally to the EIS technique, the rapid chloride penetrability test (RCPT) was performed on mortar mixtures after approximately 90 days of curing. This test, described by the ASTM C1202 standard $^{10}$, is one of the most widely used methods for determining the penetration of chloride ions, and is used to evaluate concrete durability. The RCPT test consists of estimating the ability of concrete to resist chloride penetration by obtaining the electrical charge and ranking the material according to Table 1.

Compressive strength tests were also performed on all the mixture samples 28 days after curing, which is the minimum curing time recommended for concrete structures. These tests were performed according to the standard ASTM C109 ${ }^{11}$.

\subsection{Materials}

The materials used in mortar samples were Ordinary Portland cement (OPC), industrial metakaolin, No. 30 silica sand obtained from a local quarry (see Figure 2), and water obtained from the local water supply. Table 2 shows the chemical composition of metakaolin, obtained from $\mathrm{X}$-Ray Fluorescence (XRF) and from the manufacturer's specifications.

The morphological aspects of the cementing anhydrides are shown in Figure 3, which shows scanning electronmicrographs (SEM) for ordinary Portland cement (OPC) and metakaolin (MK) samples used in the study. Well-gradated groups of coarse and fine powder with angular forms were observed in both materials. From the photographs it can be

Table 1. ASTM C1202 ${ }^{10}$ ranking (charge measured vs. concrete chloride penetrability).

\begin{tabular}{cc}
\hline Charge passed $(\mathbf{C})$ & Chloride permeability \\
\hline$>4000$ & High \\
$2000-4000$ & Moderate \\
$1000-2000$ & Low \\
$100-1000$ & Very low \\
$<100$ & Negligible \\
\hline
\end{tabular}

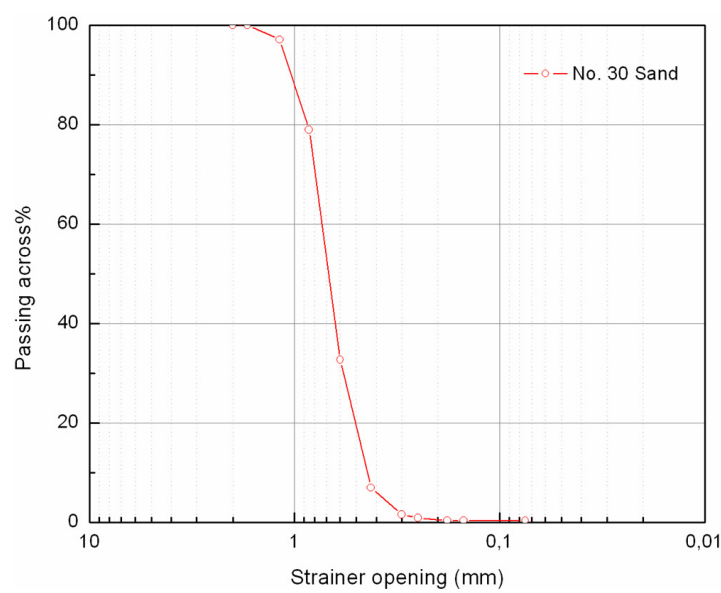

Figure 2. Particle size distribution (No. 30 sand). 


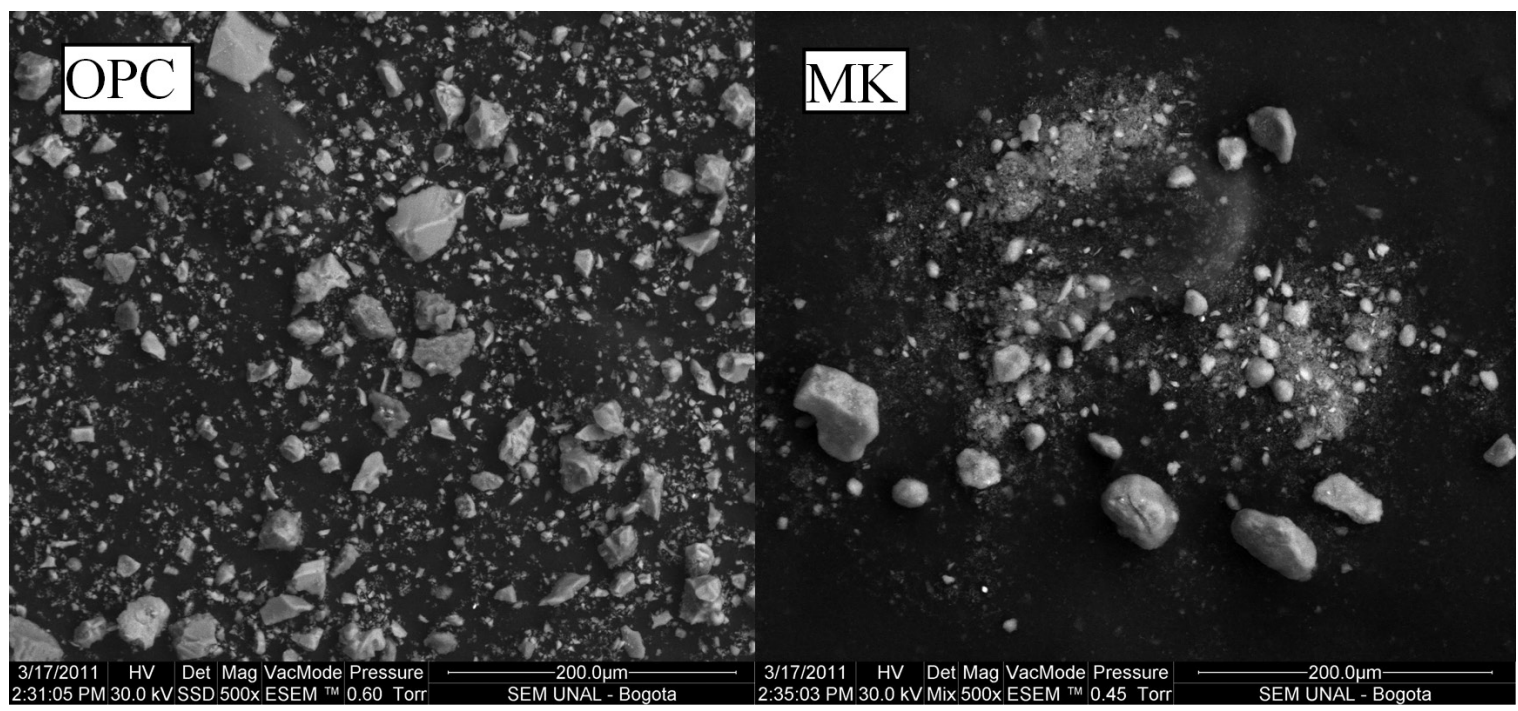

Figure 3. Scanning electron-micrograph of both cementitious materials (OPC and MK).

Table 2. Metakaolin chemical composition obtained from X-Ray Fluorescence (XRF) and from the manufacturer's specifications.

\begin{tabular}{ccc}
\hline $\begin{array}{c}\text { Element and/or } \\
\text { compound }\end{array}$ & $\begin{array}{c}\text { XRF } \\
\text { (measured) }\end{array}$ & $\begin{array}{c}\text { Manufacturer } \\
\text { Specifications (\%) }\end{array}$ \\
\hline $\mathrm{SiO}_{2}$ & $51.95 \%$ & $51-53$ \\
$\mathrm{Al}_{2} \mathrm{O}_{3}$ & $42.09 \%$ & $42-44$ \\
$\mathrm{CaO}$ & $0.08 \%$ & $<0.20$ \\
$\mathrm{~K}_{2} \mathrm{O}$ & $1.07 \%$ & $<0.40$ \\
$\mathrm{Fe}_{2} \mathrm{O}_{3}$ & $1.55 \%$ & $<2.20$ \\
$\mathrm{TiO}_{2}$ & $2.13 \%$ & $<3.0$ \\
$\mathrm{MgO}$ & $0.20 \%$ & $<0.10$ \\
$\mathrm{Na}_{2} \mathrm{O}$ & $0.09 \%$ & $<0.05$ \\
$\mathrm{P}_{2} \mathrm{O}_{5}$ & $0.15 \%$ & $<0.20$ \\
$\mathrm{SO}_{4}$ & - & $<0.5$ \\
$\mathrm{~S}$ & $218 \mathrm{ppm}$ & \\
$\mathrm{Zr}$ & $134 \mathrm{ppm}$ & \\
$\mathrm{Cr}$ & $92 \mathrm{ppm}$ & \\
$\mathrm{Zn}$ & $57 \mathrm{ppm}$ & \\
$\mathrm{Ni}$ & $64 \mathrm{ppm}$ & \\
\hline
\end{tabular}

seen that OPC exhibited a maximum particle size of $70 \mu \mathrm{m}$ and an average size of $25 \mu \mathrm{m}$, while the MK had maximum and average particle sizes of $50 \mu \mathrm{m}$ and $20 \mu \mathrm{m}$, respectively, indicating a fine powder with a high specific surface, and therefore a high reactivity.

Figure 4 shows the X-Ray diffraction spectra of the anhydrous cementing materials. The OPC showed a high number of minerals with well-defined crystalline structures in contrast to the metakaolin, which showed a glassy structure. The low crystalline and amorphous characteristics of the MK were the result of the dehydroxylation of kaolinite; however, the presence of kaolinite was detected in addition to the peak associated to quartz, indicating that the MK fabrication parameters used for this batch (temperature and calcination time) did not result in its total consumption.
This condition can affect the pozzolanic properties of MK because it would result in a non-reactive material.

The cementitious material mixtures (OPC and MK) developed in this study were blended with sand in a proportion of $1: 2.25$ ( $\mathrm{cm} / \mathrm{sand})$. The water to cementitious material ratio (w/cm by weight) under study were 0.5 and 0.6 , while the percentages of MK used were of $0 \%$ and $20 \%$. The later has been determined as an optimal percentage in previous research ${ }^{12}$, which was compared with control samples that had no mineral admixtures $(0 \% \mathrm{MK})$. Figure 5 shows conceptually the experimental design and Table 3 summarizes the sample mix design.

\subsection{Test procedures}

Because the concrete testing procedures with EIS are not normalized, several protocols published in high-impact factor journals were analyzed. Cylindrical mortar reinforced samples were cast following the approach of Ismail \& $\mathrm{Ohtsu}^{5}$, where mortar specimens were $0.10 \mathrm{~m}$ high and $0.05 \mathrm{~m}$ in diameter, with a 0.10 -m-long, 0.0055 -m-diameter steel bar embedded in the center. The mortar samples were cylindrical to assure a radial and equidistant chloride attack on the steel bar from the outer surface. Mortar mixtures were used as an approximation of concrete samples for scaling reasons and compaction of the samples was performed on 3 layers, each tamped with a $1 / 2$ " flat bar to avoid vacuum spots and achieve maximum density of the material. The steel bars were coated with epoxy along $0.04 \mathrm{~m}$ of its length, leaving $0.06 \mathrm{~m}$ exposed. The top and bottom sides of the cylindrical sample were also coated with epoxy to ensure an exclusively radial chloride attack. A screw-thread system was adapted at the end portions of the bars to fix mounting electrical connections to the sample. Figure 6 shows schematically the samples used for EIS tests. Figure 7 shows the epoxy coating process used on the sample preparation.

The equipment used for the EIS tests was a Gill AC potentiostat from ACM Instruments with 3 output terminals, 

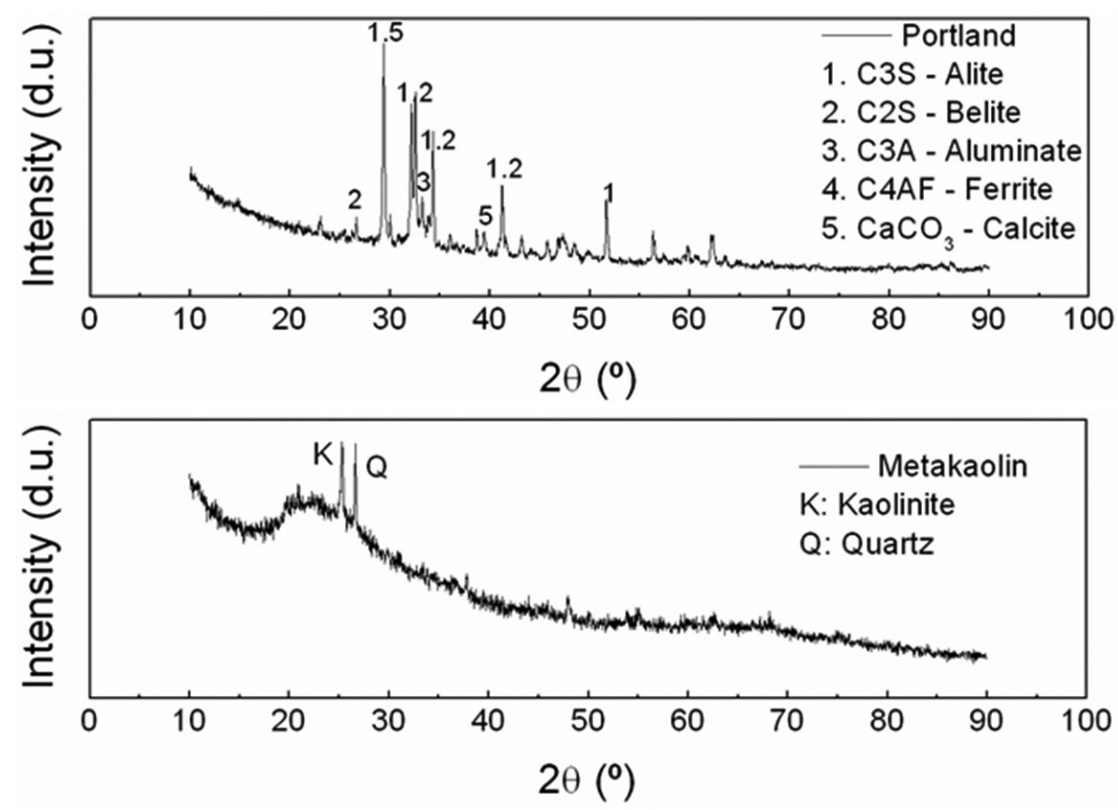

Figure 4. X-ray diffraction spectrum for both cementitious materials (OPC and MK).

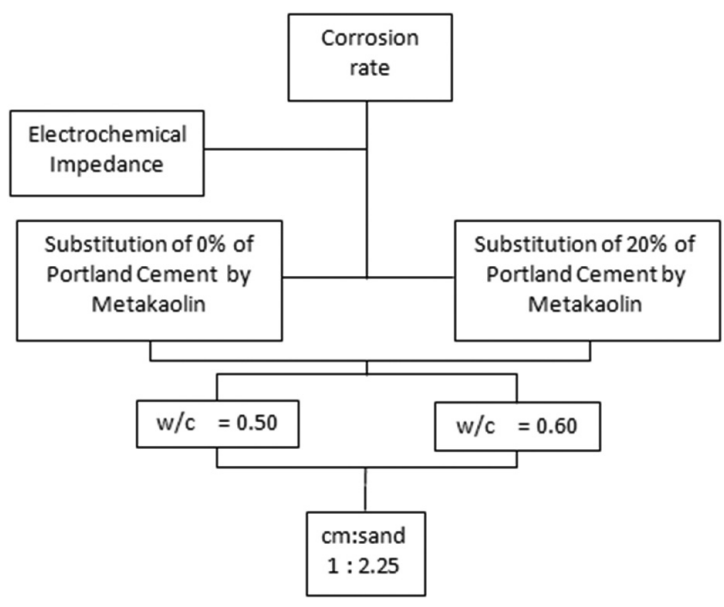

Figure 5. Conceptual experimental design.

Table 3. Design of mixtures investigated.

\begin{tabular}{cccc}
\hline Sample & \% Portland cement & \% Metakaolin & w/c \\
\hline OP0.6 & 100 & 0 & 0.6 \\
MK0.6 & 80 & 20 & 0.6 \\
OP0.5 & 100 & 0 & 0.5 \\
MK0.5 & 80 & 20 & 0.5 \\
\hline
\end{tabular}

one for the working electrode (reinforcing bar), one for the reference control electrode (calomel), and another for the auxiliary electrode or graphite contra-electrode. During the EIS tests, the samples were submerged to a depth of $0.09 \mathrm{~m}$ inside a container with a solution of $3 \% \mathrm{NaCl}$. The applied sinusoidal voltage was $10 \mathrm{mV}$ with a frequency

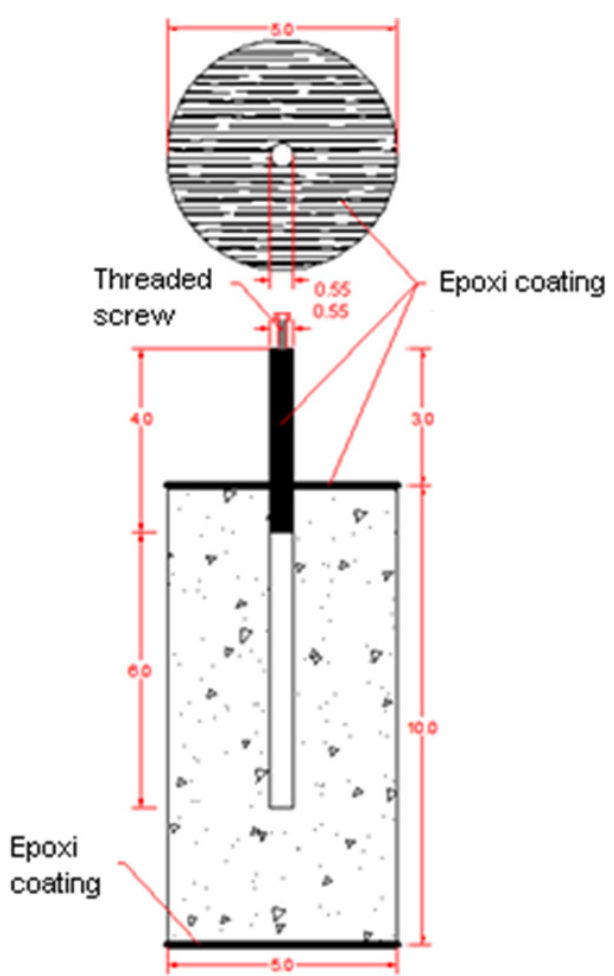

Figure 6. Experimental reinforced mortar sample (units: $\mathrm{cm}$ ).

range between $10^{-2}$ and $10^{5} \mathrm{~Hz}$. The higher frequencies (those higher than $10^{3}$ or $10^{4} \mathrm{~Hz}$, depending on how closed the pores were) reflected the electrolyte impedance through the "continuous / discontinuous pores" of the matrix. Intermediate frequencies (between $10^{0}$ and $10^{3}$ or $10^{1}$ and $10^{4} \mathrm{~Hz}$, depending on the previous range) described the 

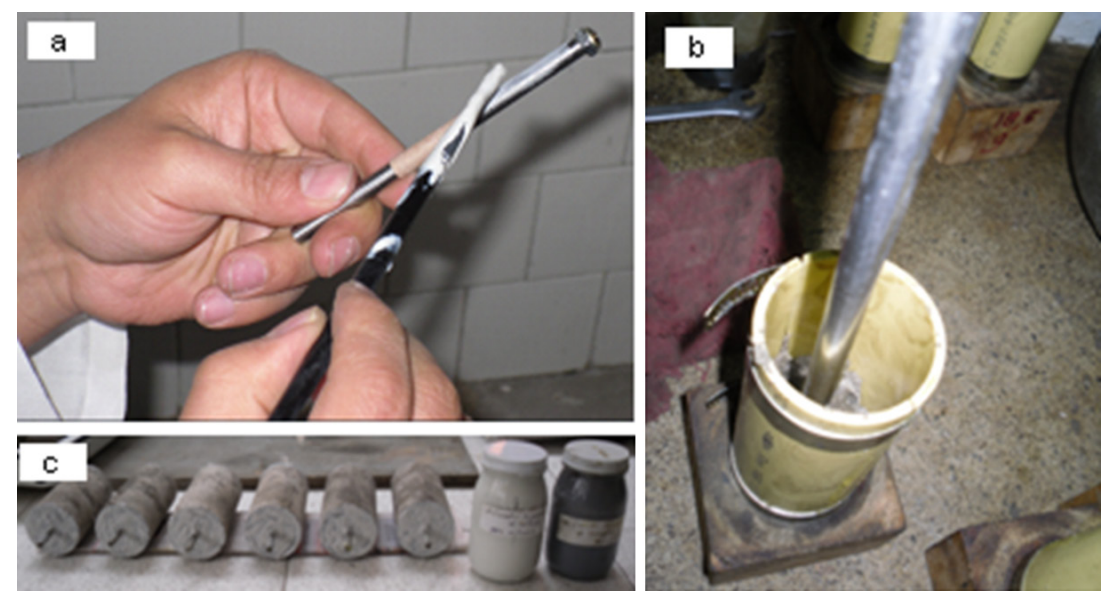

Figure 7. Preparation of reinforced concrete samples a) Epoxy coating of steel bars; b) casting of reinforced concrete samples; c) epoxy coating of mortar samples.

behavior of the blocked porosity. The lowest frequencies (those lower than $10^{\circ}$ or $10^{1} \mathrm{~Hz}$, depending on the previous range) described the charge transfer and diffusion processes at the steel-corrosion residue interface ${ }^{4,6}$.

Prior to start the impedance measurements, the samples were kept immersed in a $3 \% \mathrm{NaCl}$ solution and connected in series to an electrical circuit that induced a constant anodic potential of $100 \mathrm{mV}$ (DC) to accelerate the corrosion processes. Each sample was monitored for a minimum period of one week to distinguish the differences between subsequent measurements that occurred over a period of 495 days. 24 hours before each EIS experiment, the external anodic voltage was unplugged in order to let the samples recover their rest potential.

Standard samples were fabricated for the RCPT test with a height of $0.20 \mathrm{~m}$ and a diameter of $0.10 \mathrm{~m}$. They were then sliced into 4 equal parts measuring $0.05 \mathrm{~m}$ each. The end slices were discarded and the two central portions were extracted and coated along their entire perimeter surface with impermeable coating to avoid loss of the initial saturation conditions. The test samples were saturated according to the ASTM C1202 protocols ${ }^{10}$. For compression testing, standard cubes with edges of $0.05 \mathrm{~m}$ were tested to the point of failure.

\section{Results and Analysis}

Figures 8-12 show the Nyquist diagrams obtained at $35,80,108,133$ and 495 days after casting, and after being subjected to the conditions given in Table 4. Figure 8 indicates that samples MK0.6 and MK0.5, corresponding to those samples including MK were displaced along the real axis Z' with respect to the non-blended samples, OP0.6 and $\mathrm{OP} 0.5$, at high and intermediate frequencies. This result indicated the matrix pores' higher resistance to the solution flow. The figure also shows the formation of a semicircle, indicating the greater capacitive behavior of the blended samples that resulted in the pores' greater obstructions.

The blended samples exhibited enhanced behavior against corrosion when compared to the non-blended samples at low frequencies, indicating a passive conformation of the protective film on the bar. At this stage, samples with a
Table 4. Condition of the samples before measurement for each period of time.

\begin{tabular}{|c|c|}
\hline $\begin{array}{l}\text { Period } \\
\text { (days) }\end{array}$ & Condition \\
\hline $0-35$ & $\begin{array}{l}\text { Saturation by immersion in water for } 28 \text { days and air } \\
\text { drying for } 7 \text { days }\end{array}$ \\
\hline $35-80$ & $\begin{array}{l}\text { Saturation by immersion in a } 3 \% \mathrm{NaCl} \text { solution and } \\
\text { corrosion induced by an anodic voltage of }+100 \mathrm{mV}\end{array}$ \\
\hline $80-108$ & $\begin{array}{l}\text { Saturation by immersion in a } 3 \% \mathrm{NaCl} \text { solution and } \\
\text { corrosion induced by an anodic voltage of }+100 \mathrm{mV}\end{array}$ \\
\hline $108-133$ & $\begin{array}{l}\text { Saturation by immersion in a } 3 \% \mathrm{NaCl} \text { solution and } \\
\text { corrosion induced by an anodic voltage of }+100 \mathrm{mV}\end{array}$ \\
\hline $133-495$ & $\begin{array}{l}\text { Saturation by immersion in a saline solution (no } \\
\text { anodic voltage) }\end{array}$ \\
\hline
\end{tabular}

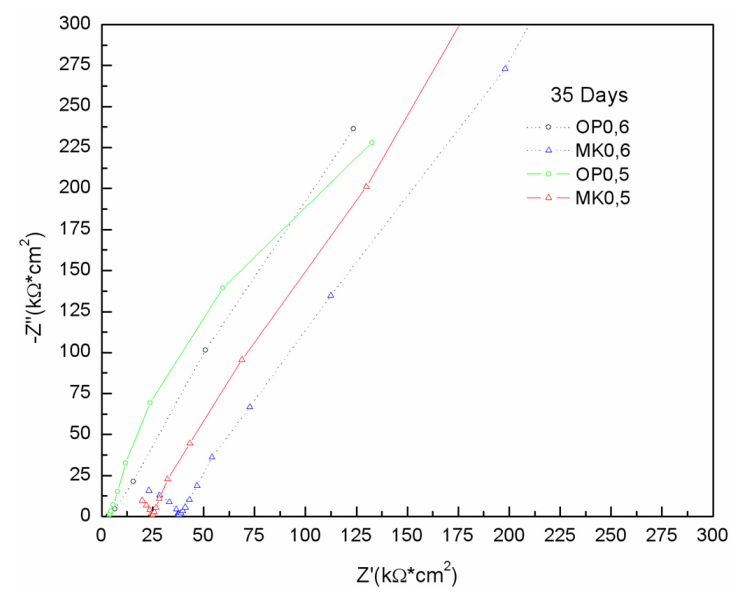

Figure 8. Nyquist diagram at 35 days.

w/c of 0.6 exhibited improved behavior over those with the same conditions but a w/c of 0.5. This finding is not in agreement with traditional concrete technology, in which a lower w/c ratio suggests that concrete will exhibit better properties. However, for this early age, these results could be due probably to the curing period or the fact that the 


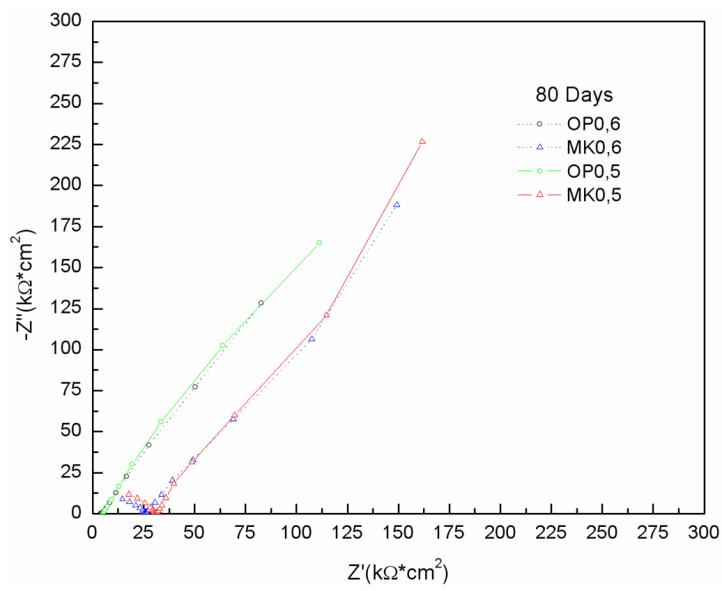

Figure 9. Nyquist diagram at 80 days.

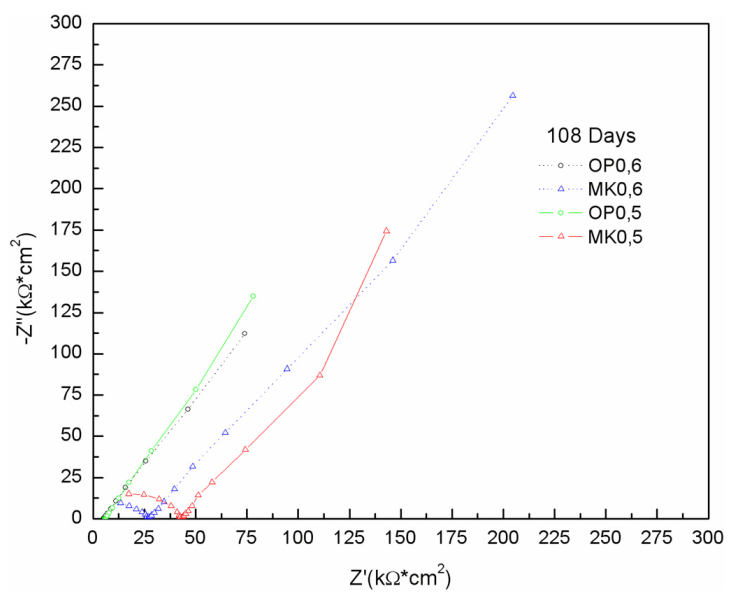

Figure 10. Nyquist diagram at 108 days.

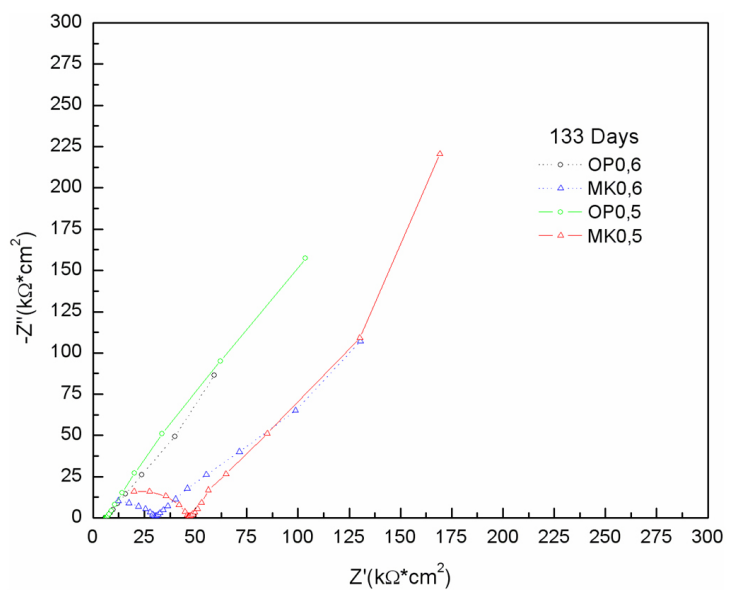

Figure 11. Nyquist diagram at 133 days.

hydration of the cementitious material was incomplete in the samples with a lower w/c ratio. The results obtained at lower frequencies corresponded to those found at high and intermediate frequencies, where the resistance to charge transfer was higher in mortars with MK and the hydroxide

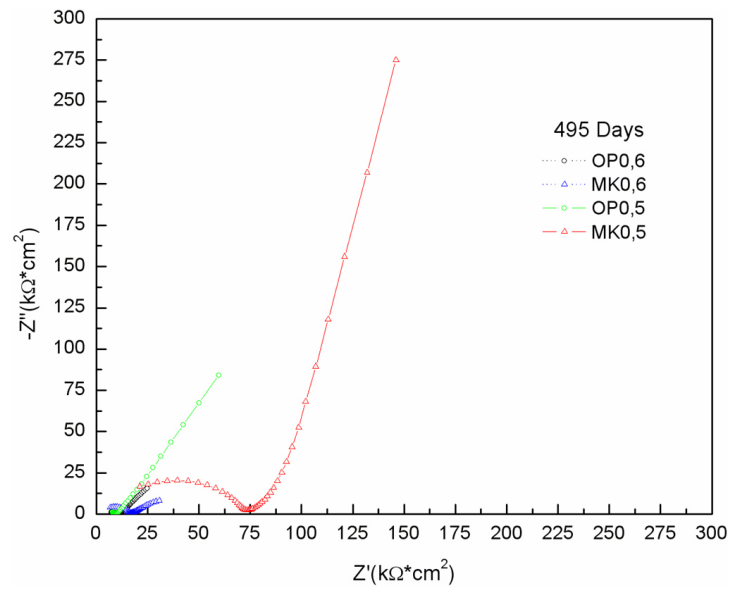

Figure 12. Nyquist diagram at 495 days.

coating layer of steel had less instability. This film should have appeared after approximately 6 days of curing according to Pérez ${ }^{6}$.

Figure 9 shows the Nyquist diagram at 80 days, following a period of immersion in a $3 \% \mathrm{NaCl}$ solution and induction with an anodic electrical voltage of $+100 \mathrm{mV}$. It is possible to note a decrease in the impedances at high and intermediate frequencies in the figure, as well as in those at low frequencies. This result occurred because the saturation of pores favored the ionic flux through the matrix, which did not occur in the first measurement at 35 days, where the samples were previously subjected to a 7-day drying period. In addition to the most noticeable impedance decrease in the blended matrices, the samples exhibited a higher resistance to the penetration of aggressive ions at this age, resulting in greater protection of the steel. It is worth noting that the samples with a w/c of 0.5 exhibited a better matrix behavior than the corresponding sample with a ratio of 0.6 , indicating that the cementitious material that had not hydrated during the initial curing had now completed its curing in accordance with the conventional concrete technology.

The samples' behavior at 108 days is shown in Figure 10. An increase in the matrix impedance occurred at higher and intermediate frequencies, which was more noticeable in samples blended with a w/c of 0.5 and was a result of the cementitious material's continuous hydration. However, while the bar embedded in the OPC matrix with a w/c of 0.5 exhibited improved protection compared to the corresponding sample with a w/c of 0.6 , the bar embedded in the MK sample at a w/c of 0.5 exhibited greater instability of its passive film than that a $\mathrm{w} / \mathrm{c}$ of 0.6. This phenomenon cannot be attributed to the effects of higher penetration of the aggressive agents at the interface because the MK matrix with a w/c of 0.5 had improved properties in this respect. Instead, this result was attributed to chemical effects because the MK reactions that favored the closing of pores via hydroxide absorption produced instabilities in the bar's passive coating film. Despite these results, the blended samples exhibited improved behavior along the entire frequency range compared to those containing only OPC. 
Figure 11 shows how the MK matrices continue to exhibit better ionic resistance compared to those with OPC at 133 days. However, bar's coating film in the matrices with a w/c of 0.5 showed improved protection. It is noteworthy that the MK sample with a w/c of 0.6, despite their matrices' superior behavior to that of the OPC sample, exhibited lower impedances at the steel interface than the equivalent OPC samples. The aforementioned discussion explains this result because the MK absorbed hydroxides to form the silicates obstructing the pores, which produced a reduction of the $\mathrm{pH}$ that altered the bar's passive coating film and produced higher porosities in the sample with a w/c of 0.6 than in the sample with a w/c of 0.5 . This porosity resulted in higher instability at the interface due to the presence of aggressive agents, which were chlorides in this case, and also explained why the same behavior did not occur in the bar embedded in the blended sample with a w/c of 0.5 . While this sample also produced a $\mathrm{pH}$ reduction, it closed its pores by having a lower w/c ratio, which prevented the penetration of chlorides. These results validate those of earlier studies showing that corrosion rates were proportional to the $\mathrm{Cl}^{-}$/ $\mathrm{OH}^{-}$ratio $^{13}$.

Figure 12 shows that the results obtained for the samples at 133 days were corroborated by the results at 495 days after a long period of immersion in a $\mathrm{NaCl}$ solution. The matrices showed improved impedances at higher and intermediate frequencies, with the exception of MK at a w/c of 0.6, which was slightly affected, while its corresponding sample at a w/c of 0.5 showed enhanced behavior along the entire frequency range and increased impedances at its matrix (with higher resistance to the ion flux) and at the interface of the bar (creating a protective film). This sample was the only one exhibiting such behavior. The importance of low w/c ratios was noted because despite the decreased protection in the OPC sample with a w/c of 0.5 , it became clear that this sample was superior to the OPC sample with a w/c of 0.6 . The current density for all the mixtures investigated was also calculated during this time. Results are shown in Figure 13, where it can be confirmed that the statements given from the Nyquist plots are in concordance with those results.

It was concluded from these results that the stability of the passive coating film was compromised in a cyclical way because two reactions occurred at the interface, one that tended to generate a protective film and another that tended to consume it, with the extent of the corrosion depending on which of these reactions occurred the most ${ }^{6}$. In the MK

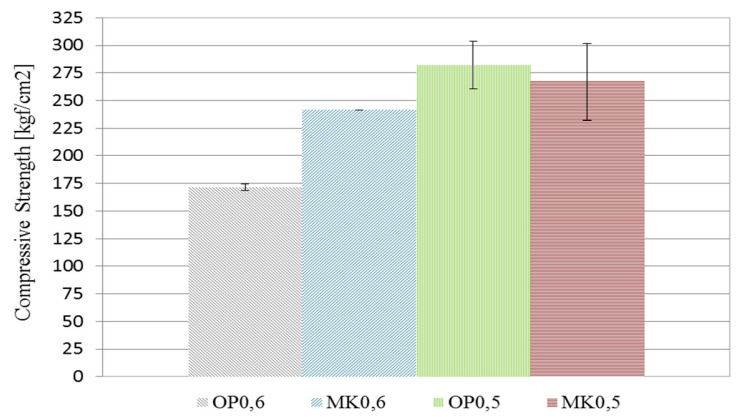

Figure 13. Current density of mortar samples measured at 495 days. sample with a w/c of 0.5 , the reaction that consumed the film occurred primarily at early ages due to its initial decreasing in $\mathrm{pH}$ and relatively high degree of porosity, but the film was again generated at later ages due to the significant reduction of pores, which in turn enabled the creation of the passive film. The MK sample with a w/c of 0.6 exhibited the opposite behavior: the rapid hydration of the cementitious material initially provided it with enhanced properties, which were subsequently affected by its higher porosity combined with a reduction in $\mathrm{pH}$.

RCPT permeability tests for chlorides were performed after a curing period of 90 days to complement the EIS tests. The results confirmed the previous findings that the impedances on the matrices with $\mathrm{MK}$ were superior to those with OPC. The chloride penetrability of the blended samples was "very low" according to the protocols of the ASTM C1202 ${ }^{10}$ standard and "moderate" in non-blended mixtures. The results are summarized in Table 5. However, this test did not allow for the assessment of the bar's behavior, as was the case with the EIS because better impedances in the matrix were not necessarily reflected in the bars.

The results of the compressive strength tests performed after 28 days of curing are shown in Figure 14, which indicates that the MK samples exhibited adequate strength at that age. The MK samples with a w/c of 0.6 had much greater strength than those containing only OPC and were slightly lower than those with OPC at a w/c of 0.5. Similarly, and as expected, the strength increased as the water-cement ratio decreased.

\section{Conclusions}

The EIS tests performed in this study suggest that reinforcing steel embedded in mortar mixtures with a low w/c of 0.5 and with MK of $20 \%$ OPC exhibited much less

Table 5. Mortar chloride penetrability according to ASTM C1202 10 tests (Table 1).

\begin{tabular}{ccc}
\hline Sample & $\begin{array}{c}\text { Adjusted average } \\
\text { charge passed (C) }\end{array}$ & $\begin{array}{c}\text { Chloride } \\
\text { Penetrability }\end{array}$ \\
\hline OP0.6 & 3977 & Moderate \\
MK0.6 & 343 & Very low \\
OP0.5 & 2426 & Moderate \\
MK0.5 & 266 & Very low \\
\hline
\end{tabular}

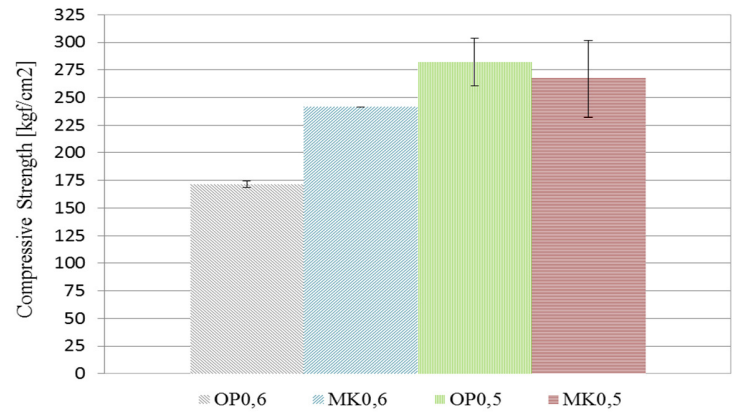

Figure 14. Strength results (ASTM C349). 
corrosion than samples containing only OPC and samples with a w/c of 0.6. On the contrary, the latter case was the least effective at protecting the bar, with values below those observed in the OPC control samples. This behavior was related to the $\mathrm{Cl}^{-} / \mathrm{OH}^{-}$ratio because the $\mathrm{MK}$ absorbed $\mathrm{OH}^{-}$in the blended mixtures, reducing the $\mathrm{pH}$. However, an important decrease in porosity occurred in the samples with low w/c ratios and prevented the intake of $\mathrm{Cl}^{-}$such that the bar was not affected in the long term. The same did not occur in samples with a w/c of 0.6 because the ions contributed to a decrease in $\mathrm{pH}$, resulting in greater deterioration of the bar. This behavior held true for the $\mathrm{cm} /$ sand ratio because its decrease generated lower capacity in the matrix and the porosity increased. The same behavior occurred in the sample with a w/c of 0.6 .

The penetration of chlorides in the mortar mixtures blended with $20 \%$ MK exhibited "very low" permeability

\section{References}

1. Jones D. Principles and Prevention of Corrosion. Prentice Hall; 1996.

2. Thompson N, Yunovich M and Dunmire D. Cost of corrosion and corrosion maintenance strategies. Corrosion Reviews. 2007; 25(3-4):247-262. http://dx.doi.org/10.1515/ CORRREV.2007.25.3-4.247

3. Kosmatka S, Kerkhoff B and Panarese W. Design and Control of Concrete Mixtures. Portland Cement Association; 2003.

4. Song G. Equivalent Circuit Model for AC Electrochemical Impedance Spectroscopy of Concrete. Cement and Concrete Research. 2000; 30(11):1723-1730. http://dx.doi.org/10.1016/ S0008-8846(00)00400-2

5. Ismail $\mathbf{M}$ and Ohtsu $\mathbf{M}$. Corrosion rate of ordinary and high-performance concrete subjected to chloride attack by AC impedance spectroscopy. Construction and Building Materials. 2006; 20(7):458-469. http://dx.doi.org/10.1016/j. conbuildmat.2005.01.062

6. Pérez T. Aplicación de la técnica Espectroscopía de Impedancia Electroquímica (EIS) en el estudio de la corrosión del acero de refuerzo embebido en concreto. Autonomous University of Campeche, Corrosion Program of the Gulf of Mexico; 2002.

7. Giannotti da Silva F and Libardi JB. A study of steel bar reinforcement corrosion in concretes with SF and SRH using Electrochemical Impedance Spectroscopy. Materials to chlorides during the RCPT tests, while the patterns in the samples containing only OPC were "moderate". However, these results were only an approximation to what occurred at the interface because they neglected the behavior of the bar, while electrochemical technique employed in this study did not.

The use of MK at 20\% helped maintain the concrete's 28-day compressive strength exhibited in the non-blended samples. However, tests should be performed at much older ages under the conditions corresponding to those maintained for the electrochemical tests because the impedance decreased in MK samples with a w/c of 0.6.

\section{Acknowledgements}

The authors acknowledge the financial support provided by the National University of Colombia for Project 13309.

Research. 2006; 9(2):209-215. http://dx.doi.org/10.1590/ S1516-14392006000200018

8. Song HW and Saraswathy V. Analysis of corrosion resistance behavior of inhibitors in concrete using Electrochemical Techniques. Metals and Materials International. 2006; 12(4):323-329. http://dx.doi.org/10.1007/BF03027549

9. Feliu V, González JA, Andrade C and Feliu S. Equivalent circuit for modeling the steel-concrete interface. I. experimental evidence and theoretical predictions. Corrosion Science. 1998; 40(6):975-993. http://dx.doi.org/10.1016/S0010938X(98)00036-5

10. American Society for Testing and Materials - ASTM. C1202-2005: Standard Test Method for Electrical Indication of Concrete's Ability to Resist Chloride Ion Penetration. West Conshohocken: ASTM International; 2005.

11. American Society for Testing and Materials - ASTM. C109-1997: Standard Test Method for Compressive Strength of Hydraulic Cement Mortars (Using 2-in. or [50-mm] Cube Specimens. West Conshohocken: ASTM International; 1997.

12. Torres J, Mejía de Gutiérrez R and Puertas F. Effect of kaolin treatment temperature on mortar chloride permeability. Materiales de Construcción 2007; 57(285):61-69.

13. Thangavel K and Rengaswamy NS. Relationship between chloride/hydroxide ratio and corrosion rate of steel in concrete. Cement and Concrete Composites. 1998; 20(4):283-292. http:// dx.doi.org/10.1016/S0958-9465(98)00006-7 